\title{
Evaluation of rebreathed air in human nasal cavity with N95 respirator: a CFD study
}

\author{
Jian Hua Zhu' ${ }^{1 *}$, Shu Jin Lee ${ }^{2}$, De Yun Wang ${ }^{3}$ and Heow Pueh Lee ${ }^{1}$ \\ ${ }^{1}$ Department of Mechanical Engineering, National University of Singapore, Singapore \\ ${ }^{2}$ Department of Surgery, National University Health System, Singapore \\ ${ }^{3}$ Department of Otolaryngology, National University of Singapore, Singapore
}

\begin{abstract}
The carbon dioxide level within N95 respirator is higher than without it, which needs a mechanical explanation. In the current study, we built a three-dimensional (3D) model of normal human nasal cavity to simulate the volume of fraction of both fresh air and respired air within the nasal cavity. The model consists of large rectangular domain outside the nasal cavity representing ambient air, human nasal cavity and partial of the pharynx. Two cases were simulated. Case I refers to a human face with a N95 respirator onto human face, and case II refers to a human face without a respirator. Respiration cycles of both low (7.5 L/min) and medium ( $15 \mathrm{~L} / \mathrm{min}$ ) flow rates were simulated using Low Reynolds Number $\mathrm{k}-\omega$ turbulent model. All the air entered the external box during inspiration was assumed to be fresh air, and entered the pharynx during expiration was assumed to be respired air. The results show that above $60 \%$ of inspired air is respired air in case I compared to less than $1.2 \%$ in case II. During expiration, the volume of fraction (VOF) of respired air in both cases was above $95 \%$. The streamlines at peak inspiration were relatively smooth while entering the cavity in both cases; while at peak expiration large vortex was observed within the air space between human face and respirator in case I. In conclusion, the N95 respirator trapped respired air within the respirator which increased the VOF of respired air during inspiration. This might be one of the major contributors to elevated carbon dioxide level while wearing N95 respirator.
\end{abstract}

\section{Introduction}

Wearing masks and respirators would increase user burden due to the low permeability especially for respirator. Wearing an N95 respirator was reported to cause headaches among healthcare providers [1]. N95 respirator and surgical facemask also causes different heart rate and subjective perception of discomfort [2]. The physiology for these phenomena is not fully understood. One possible reason is due to the change of physical conditions around the respirators and masks. For example, due to slowing down of heat and substance dissipation, both N95 respirator and surgical facemask cause variations of microclimates around the masks [3]. Wearing respirator or mask would affect inhaled gas concentrations and respiratory resistances as well [4]. Particularly, wearing the respirator elevated the carbon dioxide level while decreased oxygen level within the respirator which may be the cause of subjective complaints for wearing respirators [5]. The increased partial carbon dioxide level also tends to affects breathing patterns and heart rate variability [6]. However, the reason for elevated carbon dioxide level within respirator and mask is unknown. One possibility is that the expired airflow, with higher concentration of carbon dioxide, is trapped around the masks and re-enter the respiratory system during the next respiration cycle.

In the recent few decades computational fluid dynamics (CFD) simulation has been proved to be a convenient tool to investigate airflow patterns in human nasal cavity [7-10]. However, there is yet no CFD study on substance exchange between human upper airway and external environment while wearing respirator or mask. Therefore, in the current study we carried out CFD simulations to investigate how the respired air is dissipated during respiration while wearing/not wearing N95 respirator as shown in the rest of this manuscript.

\section{Materials and methodology}

Computerized tomography (CT) scans of a 47 year old female subject with normal nasal cavity morphology was acquired in this study. The same process of 3-dimensional (3D) model reconstruction and discretization of human nasal cavity as our previous study was carried out using MIMICS (version 12.1, Materialise n.v., Leuven, Belgium) and HYPERMESH (version 10.0, Atlair Engineering, Inc., MI, USA) [11]. As shown in Figure 1, there were two nasal models built. In case $\mathrm{I}$, the 3D model of an N95 filtering facepiece respirator without an exhale valve (model 3M 8210, referred to as N95 respirator hereafter)

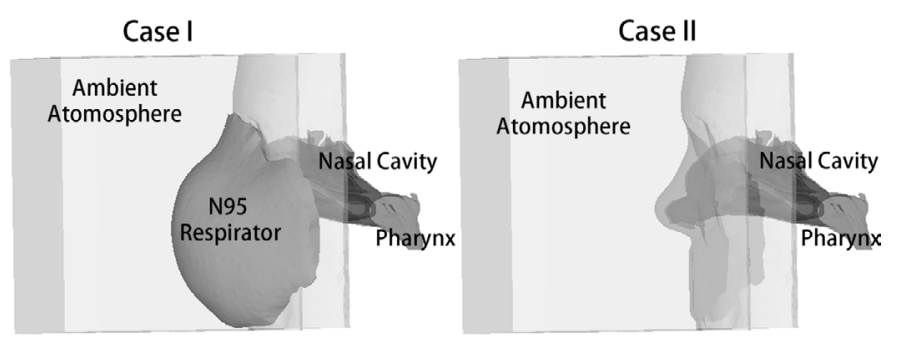

Figure 1. 3D models of human nasal cavity in cases I and II.

Correspondence to: Dr. Jian Hua Zhu, Department of Mechanical Engineering, National University of Singapore, Faculty of Engineering, 1 Engineering Drive 2 Singapore 117576; Tel: (+65) 96326956; Fax: (+65) 67791459; E-mail: Jhzhu.me@gmail.com

Key words: $N 95$ respirator, multiphase flow, respired air, fresh air, CFD

Received: January 25, 2016; Accepted: February 26, 2016; Published: February 29, 2016 
was integrated onto the human face to mimic the effects of wearing N95 respirator. This N95 respirator model was obtained by scanning the surface of it using a 3D scanner (http://www.delcam.com/). The respirator surface was imported into HYPERMESH, relocated to a position that it is aligned with human face and the majority of its edges are resting on human face with minimal gap in between. Although there is usually air leakage through the respirator, the nasal cavity was assumed to be perfectly sealed by the respirator as a simplification to the CFD model. This is achieved by merging the edge nodes of respirator with the nearest nodes on human face manually. The mask was also assumed to be inflexible. Case II in Figure 1 shows the 3D model of human nasal cavity without N95 respirator. In both models, the nasal cavity was extended to a large rectangular box $(0.15 \mathrm{~m}$ in width, $0.14 \mathrm{~m}$ in height and $0.1 \mathrm{~m}$ in the saggital direction) representing the surrounding ambient air. Partial of the pharynx was presented on the other side of the nasal cavity. For both models, five layers of prism mesh were created near the surface of computational domain with the thickness of each layer to be $0.05 \mathrm{~mm}$ to more accurately capture the near-wall viscous effects, while the core of the domains were filled with tetrahedron elements. The size of the element was set to be 0.60 $\mathrm{mm}$ in the nasal cavity domain and the respirator domain to capture small morphological features, and $1.5 \mathrm{~mm}$ for the other domains to reduce computational cost. In total there were 2.8 million number of elements created including the ambient area, the respirator in case I (or ambient area in case II) and the nasal cavity. Grid independency of the current meshing settings has been verified in our previous study [11]. In addition, this CFD method has been previously validated by comparing numerical results with reported experimental data for other typical nasal airflow studies [12].

Instead of simulating all the air compositions, the air was simplified into two components: the fresh air that was brought into the nasal cavity from the ambient during inspiration, and the respired air that was breathed out during expiration from the pharynx. The properties of air in the material database of ANSYS FLUENT (Version 14.0, ANSYS, Inc., PA, USA) were used as fresh air. In addition, the respired air was assumed to consist of 78.04\% nitrogen, $16.00 \%$ Oxygen, $5.00 \%$ carbon dioxide and $0.96 \%$ Argon. The overall density and viscosity were calculated proportionally based on the accordingly properties of these components specified in the database. The N95 respirator in case I was simplified as porous media. The same porous properties for N95 respirator as Lei et al. [13] and Li et al. [14] were used, where porosity is 0.88 , and the viscous resistance coefficient is $1.12 \times 10^{10} 1 / \mathrm{m}^{2}$.

During simulation, the human face and surface of nasal cavity

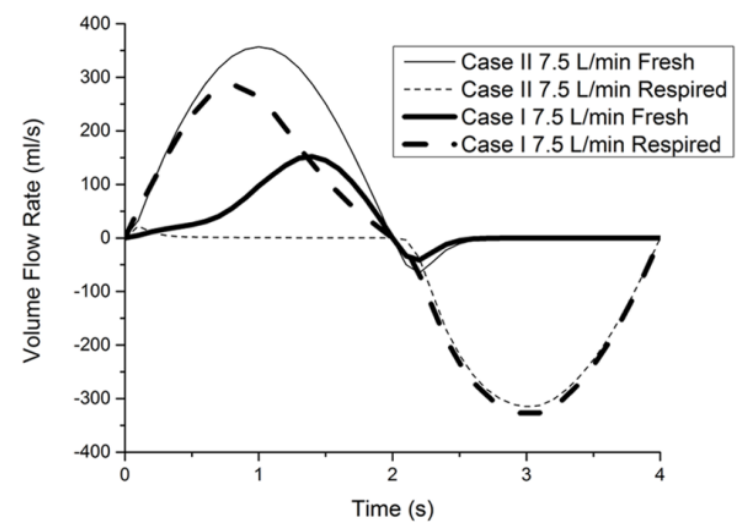

was assumed to be rigid wall. A zero gauge pressure was applied on the surface of the external box representing the surrounding ambient atmospheric condition. With pressure applied in the pharynx, airflows corresponding to ventilation rates of both $7.5 \mathrm{~L} / \mathrm{min}$ and $15 \mathrm{~L} / \mathrm{min}$ were simulated, where the volume flow rate varies sinusoidally and the periods for inspiration and expiration were both 2 seconds. The pharynx pressure applied was obtained by running the transient simulation with velocity loads (ventilation rates of $7.5 \mathrm{~L} / \mathrm{min}$ and 15 $\mathrm{L} / \mathrm{min}$ ) and measuring average cross sectional pressure at the pharynx. This measured pressure is then used as pressure boundary condition on pharynx during cyclic simulation of breathing. In addition, at inspiration the air entered the computational domain through external boundaries was assumed to be purely fresh air; at expiration, the air entered the pharynx was assumed to be purely respired air. As a multiphase problem, the mixture model in FLUENT was used to govern the two phases (fresh air and respired air). The airflow was assumed incompressible and Newtonian. Low Reynolds Number k- $\omega$ turbulent model was used to simulate all the laminar, transitional and turbulent airflow phases. At the pharynx, the turbulent length scale was set to be $0.63 \mathrm{~mm}$ based on its hydraulic diameter, and the turbulent intensity was fixed at $4 \%$. On the external boundary, the turbulent length scale was set to be $31 \mathrm{~mm}$ according to its large hydraulic diameter and the turbulent intensity was $1 \%$ since the velocity on the boundary is almost static. For each model, with the above applied boundary conditions, three cycles of respiration were simulated to ensure convergence of cyclic transient flow and the results from the last cycle were utilized for analysis. The commercial software ANSYS FLUENT was used to solve all the continuity, Navier-Stokes turbulent, multiphase and porous equations. In FLUENT, the solver was chosen as pressure-based with PISO coupling algorithm. Standard discretization method was used for pressure and first order upwind was chosen for the other variables such as momentum, turbulent kinetic energy and specific dissipation rate. Time step was set to be $0.05 \mathrm{~s}$ with 80 steps for each breathing cycle. Commercial software ENSIGHT (Version 9.0.3(a), CEI, Inc., North Carolina, USA) was used for post-processing the data generated from FLUENT.

\section{Results and discussion}

Figure 2 shows the airflow rate of fresh air and respired air through the nostrils. In Case II, the majority of the inspirational airflow is fresh air, while during expiration is respired air. In Case I, however, at the beginning of inspiration the majority of the inspirational air is respired air, while near the end of inspiration the flow rate of inspired fresh air

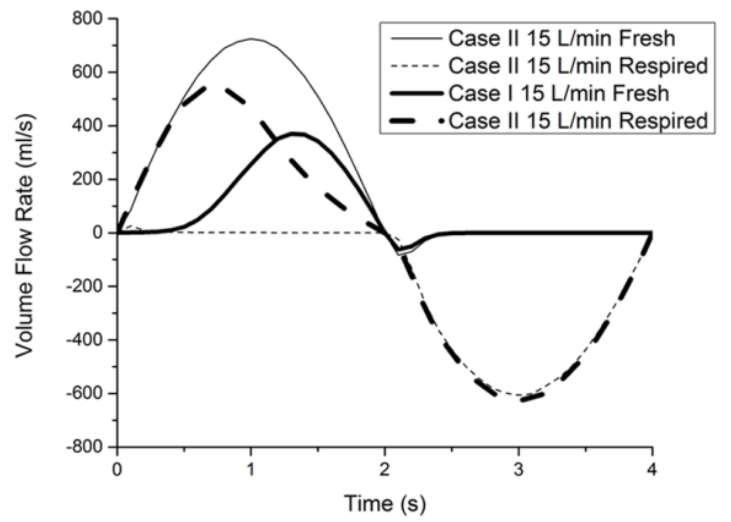

Figure 2. Volume flow rate of fresh air and respired air during respiration in both cases I and II. 
increased to be slightly larger than respired air. During expiration in Case I, the flow rate of fresh air passed through nasal valve region is minimal. The proportion of fresh air/inspired air during respiration is similar between ventilation rate of $15 \mathrm{~L} / \mathrm{min}$ and $7.5 \mathrm{~L} / \mathrm{min}$. The flow rates of fresh air and respired air through nostrils were then integrated over the whole period of simulated breathing cycle. In total, at both ventilation rates of $15 \mathrm{~L} / \mathrm{min}$ and $7.5 \mathrm{~L} / \mathrm{min}$, during the 2 seconds inspiration above $60 \%$ of the inspired air in Case I is respired air compared to less than $1.20 \%$ in Case II; while during the 2 seconds expiration, the proportion of respired air is above $95 \%$ in both cases I and II. This finding suggests an increase of carbon dioxide level and decrease of oxygen level in nasal cavity with respirator due to the rebreathed air, which is consistent with Laferty and Mckay's finding.

Figure 3 (Videos 1,2,3 and 4) shows the volume of fraction (VOF) of respired air in a saggital cross section of nasal cavity at beginning, peak and end of inspiration/expiration. The color in blue represents the volume occupied by pure fresh air, while the red color represents the volume occupied by pure respired air. The color in-between represents a mixture of fresh and respired air. At the beginning of inspiration, in case II, the cavity was filled with respired air with a mixture air stream straightly extending from the ambient to the nostril; while in case I, the respired air expanded alongside the mask to the region outside of the mask. From inspirational peak to inspirational end/beginning of expiration, the whole model was filled with fresh air in case II. However, in case I the cavity and nearby ambient region was still filled

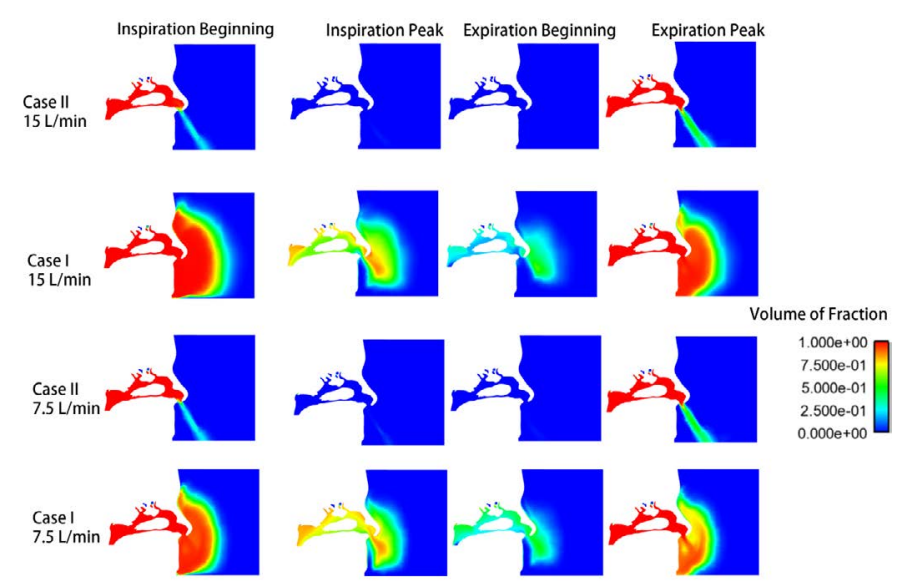

Figure 3. Volume of fraction of respired air at beginning/peak of inspiration and expiration in both cases I and II.
Video 2. 7.5 porous.mpg

Video 3. 15.mpg.

Video 4. 15 porous.mpg.

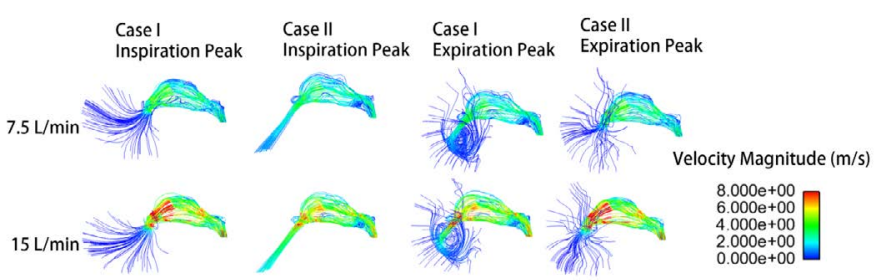

Figure 4. Streamlines at peak inspiration/expiration in both cases I and II.

with mixture of fresh/respired air with decreasing tendency of VOF of respired air with time. At peak expiration, the respired air breathed out from the pharynx was directly brought into the ambient alongside the nasal vestibule in case II. In case I, due to the existence of N95 respirator the respired air was dissipated outside of the respirator at a much slower speed. This phenomenon could be more clearly observed 
in the supplemented videos (7.5.mpg, 7.5porous.mpg, 15.mpg and 15porous.mpg). The distribution patterns of respired air between ventilation rates of $7.5 \mathrm{~L} / \mathrm{min}$ and $15 \mathrm{~L} / \mathrm{min}$ are similar. As the air inside respirator dissipates much slower than without respirator, so does the heat and vapor exchange between the respired air and fresh air. This slowed dissipation causing difference in microclimate between interior and exterior of the respirator $[2,15]$, which in turn affects the wearer's respiration and subjective sensation $[6,16]$.

Figure 4 shows the streamlines at peak inspiration/expiration in both cases. In case II, peak inspiration, the streamlines are more converged from the ambient region into the cavity. While in Case I, the streamlines arise along the whole surface of respirator into the cavity. At peak expiration, case II, the streamlines exited from the cavity directly, though with a little curvature. While in case I, large vortex was observed in the space between respirator and human face, preventing the respired air from exiting the respirator. This phenomenon explains the reason for the elevated VOF of respired air during inspiration. The simulation captured the difference of flow streamlines within the respirator in different directions such as peak inspiration and peak expiration. It is reasonable to observe such a difference as during expiration, the airflow with relatively high kinetic energy right hit on the respirator (simplified as porous media) and bounded back to form vortex within the respirator, while during inspiration the upstream airflow outside the respirator is much slower which allows the streamline to peacefully penetrate the porous media and enter the cavity.

\section{Conclusion}

In conclusion, through the current CFD simulation of multiphase flow, and by simplifying the respirational air as fresh air and respired air, we successfully observed elevated volume of fraction of respired air during inspiration. In this simplified model above $60 \%$ of respired air reentered the nasal cavity during the consecutive respirational cycle. This study also proves that CFD is a useful tool to provide reasonable explanation for experimental findings.

\section{Acknowledgement}

The authors would like to acknowledge the support by the Academic Research Grant (CDPHRG/12NOV001) from the Ministry of Health, Singapore.

\section{Conflict declaration}

The authors declare that none of the authors has any conflict of interest in this manuscript.

\section{References}

1. Lim ECH, Seet RCS, Lee KH, Wilder-Smith EPV, Chuah BYS, et al. (2006) Headaches and the N95 face-mask amongst healthcare providers. Acta Neurologica Scandinavica 113: 199-202. [Crossref]

2. Li Y, Tokura H, Guo YP, Wong AS, Wong T, et al. (2005) Effects of wearing N95 and surgical facemasks on heart rate, thermal stress and subjective sensations. Int Arch Occup Environ Health 78: 501-509. [Crossref]

3. Hayashi C, Tokura H (2004) The effects of two kinds of mask (with or without exhaust valve) on clothing microclimates inside the mask in participants wearing protective clothing for spraying pesticides. Int Arch Occup Environ Health 77: 73-78. [Crossref]

4. Sinkule EJ, Powell JB, Goss FL (2013) Evaluation of N95 Respirator Use with a Surgical Mask Cover: Effects on Breathing Resistance and Inhaled Carbon Dioxide. Ann Occup Hyg 57:384-398. [Crossref]

5. Laferty EA, McKay RT (2006) Physiologic effects and measurement of carbon dioxide and oxygen levels during qualitative respirator fit testing. J Chem Health Safety 13: $22-28$.

6. Pöyhönen M, Syväoja S, Hartikainen J, Ruokonen E, Takala J (2004) The effect of carbon dioxide, respiratory rate and tidal volume on human heart rate variability. Acta Anaesthesiol Scand 48: 93-101.

7. Kim SK, Na Y, Kim J-I, Chung S-K (2013) Patient specific CFD models of nasal airflow: Overview of methods and challenges. J Biomech 46: 299-306. [Crossref]

8. Zang H, Liu Y, Han D, Zhang L, Wang T, et al. (2012) Airflow and temperature distribution inside the maxillary sinus: A computational fluid dynamics simulation. Acta Otolaryngol 132: 637-644. [Crossref]

9. Pless D, Keck T, Wiesmiller K, Rettinger G, Aschoff AJ, et al. (2004) Numerical simulation of air temperature and airflow patterns in the human nose during expiration. Clin Otolaryngol Allied Sci 29: 642-647. [Crossref]

10. Zhu JH, Lee HP, Lim KM, Gordon BR, Wang DY (2012) Effect of accessory ostia on maxillary sinus ventilation: A computational fluid dynamics (CFD) study. Respir Physiol Neurobiol 183: 91-99. [Crossref]

11. Zhu JH, Lim KM, Gordon BR, Wang DY, Lee HP (2014) Effects of Anterior Ethmoidectomy, With and Without Antrotomy and Uncinectomy, on Nasal and Maxillary Sinus Airflows: a CFD Study. J Med Biol Eng.

12. Zhu JH, Lee HP, Lim KM, Lee SJ, Wang DY (2011) Evaluation and comparison of nasal airway flow patterns among three subjects from Caucasian, Chinese and Indian ethnic groups using computational fluid dynamics simulation. Respir Physiol Neurobiol 175: 62-69. [Crossref]

13. Lei Z, Yang J, Zhuang Z, Roberge R (2013) Simulation and Evaluation of Respirator Faceseal Leaks Using Computational Fluid Dynamics and Infrared Imaging. Annals of Occupational Hygiene 57: 493-506. [Crossref]

14. Li Y, Wong T, Chung J, Guo YP, Hu JY, et al. (2006) In vivo protective performance of N95 respirator and surgical facemask. Am J Ind Med 49:1056-1065. [Crossref]

15. Roberge RJ, Kim J-H, Benson S (2012) N95 Filtering Facepiece Respirator Deadspace Temperature and Humidity. J Occup Environ Hyg 9: 166-171. [Crossref]

16. Shenal BV, Radonovich LJ, Cheng J, Hodgson M, Bender BS (2011) Discomfort an Exertion Associated with Prolonged Wear of Respiratory Protection in a Health Care Setting. J Occup Environ Hyg 9: 59-64. [Crossref]

Copyright: (C2016 Zhu JH. This is an open-access article distributed under the terms of the Creative Commons Attribution License, which permits unrestricted use, distribution, and reproduction in any medium, provided the original author and source are credited. 